\title{
Conhecimento e atitudes de profissionais de enfermagem sobre higiene bucal em pacientes críticos
}

Knowledge and attitudes of nursing professionals regarding oral hygiene in critically ill patients Conocimientos y actitudes de los profesionales de enfermería sobre la higiene bucal en pacientes críticos

\section{Adriana Maria da Silva Felix ${ }^{\mathrm{I}}$, Isabela Di Tilia Alves do Amaral ${ }^{\mathrm{II}}$, Rosemeire Ângela de Queiroz Soares ${ }^{\text {III }}$, Regina Claudia da Silva Souza ${ }^{\text {IV }}$}

Resumo: Objetivo: comparar conhecimentos e atitudes dos profissionais de enfermagem atuantes em hospital público e privado sobre higiene bucal em pacientes críticos. Método: estudo transversal, realizado em duas Unidades de Terapia Intensiva Adulto, uma pública e outra privada no período entre dezembro de 2018 e janeiro de 2019 por meio de um questionário estruturado e autoaplicável. Resultados: a taxa de conhecimento dos profissionais do hospital público foi de $76,6 \%$ e a atitude foi de $62,7 \%$, enquanto no hospital privado essas taxas foram de $81,8 \%$ e $67,1 \%$, respectivamente. Houve diferença significativa quanto à existência de protocolo $(p<0,006)$, participação em treinamento $(p<0,004)$, uso de escova de dentes com cerdas macias e sucção $(p<0,001)$ no hospital privado. Conclusão: o nível de conhecimento foi mais elevado e as atitudes sobre higiene bucal foram mais positivas entre os profissionais de enfermagem do hospital privado em comparação com os profissionais do hospital público.

Descritores: Enfermagem; Conhecimentos, Atitudes e práticas em saúde; Higiene Bucal; Unidades de Terapia Intensiva; Estudos Transversais

\begin{abstract}
Objective: to compare the knowledge and attitudes of nursing professionals working in public and private hospitals on oral hygiene in critically ill patients. Method: a cross-sectional study carried out in two adult Intensive Care Units, one public and the other one private, between the period of December 2018 and January 2019, using a structured and self-administered questionnaire. Results: the knowledge rate of professionals in the public hospital was $76.6 \%$ and the attitude was $62.7 \%$, while in the private hospital, these rates were $81.8 \%$ and $67.1 \%$, respectively. There were significant differences regarding the existence of a protocol $(\mathrm{p}<0.006)$, participation in training ( $\mathrm{p}<0.004)$, and the use of a toothbrush with soft bristles and suction $(\mathrm{p}<0.001)$ in the private hospital.
\end{abstract}

\footnotetext{
${ }^{I}$ Enfermeira. Doutora em Ciências. Docente em Enfermagem na Faculdade de Ciências Médicas da Santa Casa de São Paulo, São Paulo, SP, Brasil. E-mail: adriana.felix@fcmsantacasasp.edu.br Orcid: http://orcid.org/0000-0002-3559-3729

II Bacharel em Enfermagem. Faculdade de Ciências Médicas da Santa Casa de São Paulo, São Paulo, SP, Brasil. E-mail: isabela.tilia@hotmail.com Orcid: http://orcid.org//0000-0002-2498-4521

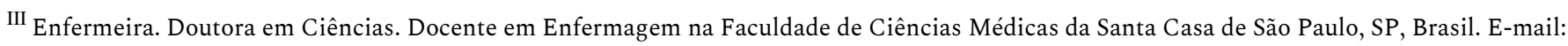
roseenfh@gmail.com Orcid: http://orcid.org/0000-0002-3752-6634

${ }^{I V}$ Enfermeira. Mestre em Enfermagem. Escola de Enfermagem da Universidade de São Paulo, São Paulo, SP, Brazil. E-mail: rclaudiasouza@uol.com.br Orcid: http://orcid.org//0000-001-6433-7700
} 
Conclusion: the knowledge level was higher, and the attitudes toward oral hygiene were more positive among nursing professionals at the private hospital compared to the professionals at the public hospital.

Descriptors: Nursing; Health knowledge, Attitudes and practices; Oral Hygiene; Intensive Care Units; Crosssectional Studies.

Resumen: Objetivo: comparar los conocimientos y actitudes de los profesionales de enfermería que laboran en un hospital público y privado sobre higiene bucal en pacientes críticos. Método: estudio transversal, realizado en dos Unidades de Cuidados Intensivos de Adultos, una pública y otra privada, entre diciembre de 2018 y enero de 2019, mediante un cuestionario estructurado y autoadministrado. Resultados: la tasa de conocimiento de los profesionales del hospital público fue del 76,6\% y la actitud del 62,7\%, mientras que en el hospital privado estas tasas fueron del $81,8 \%$ y $67,1 \%$, respectivamente. Hubo diferencia significativa con respecto a la existencia de protocolo $(\mathrm{p}<0,006)$, participación en entrenamiento $(\mathrm{p}<0,004)$, uso de cepillo de dientes con cerdas blandas $y$ succión ( $p<0,001)$ en el hospital privado. Conclusión: el nivel de conocimiento fue mayor y las actitudes sobre higiene bucal más positivas entre los profesionales de enfermería del hospital privado en comparación con los profesionales del hospital público.

Descriptores: Enfermería; Conocimientos, Actitudes y prácticas de salud; Higiene Bucal; Unidades de Cuidados Intensivos; Estudios Transversales

\section{Introdução}

A higiene bucal (HB) é um cuidado diário de enfermagem prestado ao paciente crítico, que melhora o conforto, reduz a colonização da orofaringe e contribui para a redução da incidência de infecção, por exemplo, a pneumonia associada à ventilação mecânica (PAVM). ${ }^{1}$ Guia nacional e internacional enfatizaram que o programa de HB é uma das estratégias de prevenção de PAVM.1-2 O padrão ouro de intervenção em cuidados bucais de pacientes intubados inclui a observação com ferramentas de avaliação de saúde bucal, o uso de gluconato de clorexidina $0,12 \%$ e técnicas de escovação dos dentes. ${ }^{1}$

Os pacientes críticos dependem integralmente dos cuidados prestados por profissionais de saúde, sendo que os conhecimentos e atitudes desses profissionais influenciam em sua recuperação. ${ }^{3}$ Estudos descritivos realizados em hospitais públicos e privados, que usaram questionários autoaplicáveis para a coleta de dados, descrevem que os profissionais de enfermagem apresentam conhecimento, atitudes e práticas deficientes sobre a $\mathrm{HB}$ e que fatores como conhecimento insuficiente, pouca experiência profissional, lacunas de treinamento, desconforto, medo de causar eventos adversos, tipo de Unidade de Terapia Intensiva (UTI) e pacientes pouco colaborativos contribuem para essa realidade. ${ }^{3-10}$

O primeiro passo para mudar a atitude dos profissionais de enfermagem sobre HB é conscientizá-

los de que não se trata apenas de uma medida de conforto, mais sim de uma estratégia que também 
qualifica os cuidados de enfermagem, diminui o risco de infecção e melhora os resultados dos pacientes; e isso pode ser alcançado por meio de protocolos baseados em evidência, capacitação periódica e estrutura assistencial adequada. ${ }^{11} \mathrm{~A}$ atitude positiva foi um forte fator de influência na adesão a protocolos de prevenção de infecção em hospitais na Coreia do Sul, seguido pelo apoio administrativo, tipos de hospitais e clima de segurança. ${ }^{12}$

A qualidade assistencial deve ser priorizada pelas instituições de saúde e por seus profissionais. Independente de ser público ou privado, espera-se que o serviço de saúde ofereça um cuidado seguro ao paciente. Neste seguimento, a enfermagem possui papel crucial, uma vez que representa a maior força de trabalho, prestando assistência individualizada e em tempo integral ao paciente, tendo em vista a sua rápida recuperação.

Pesquisar os conhecimentos e atitudes sobre HB dos profissionais de enfermagem que atuam em UTI de instituições públicas e privadas é fundamental, pois permite identificar oportunidades de melhoria, elaborar estratégias voltadas à qualificação da assistência e também fomentar a cultura da prática baseada em evidência. Assim, estudos que permitam conhecer a realidade sobre o conhecimento e as atitudes dos profissionais nas organizações de saúde referente às práticas em questão, contribuem para um melhor entendimento do impacto que os aspectos organizacionais e individuais exercem sobre a qualidade do cuidado. Dessa forma, o objetivo do presente estudo é comparar conhecimentos e atitudes dos profissionais de enfermagem atuantes em hospital público e privado sobre HB em pacientes críticos.

\section{Método}

O estudo transversal foi realizado em duas UTIs adulto, de duas instituições de saúde localizadas na cidade de São Paulo. A UTI do hospital público (Hospital A) possui 42 leitos, enquanto que a UTI do hospital privado (Hospital B) possui 20 leitos com perfil de atendimento a pacientes de especialidades clínicas e cirúrgicas.

A coleta de dados ocorreu no período de dezembro de 2018 a janeiro de 2019. Para atender o objeto da pesquisa, foi desenvolvido um questionário estruturado e autoaplicável baseado em guia nacional $^{1}$ e internacional ${ }^{2}$ de prevenção de pneumonia associada à assistência à saúde e publicações 
Conhecimento e atitudes de profissionais de enfermagem sobre higiene bucal em... 4

científicas sobre HB. ${ }^{3-16}$ Esse instrumento contemplou 37 itens e foi organizado em duas partes. A primeira foi composta por dez itens referentes às características sociodemográficas e profissionais, e a segunda parte foi composta por 13 itens sobre conhecimentos e 13 itens referentes às atitudes sobre HB, os quais foram apresentados em escala Likert de 4 pontos $(1=$ discordo totalmente; $2=$ discordo; $3=$ concordo; 4=concordo totalmente).

Antes da aplicação, o instrumento foi submetido à validação de conteúdo por oito enfermeiros especialistas em Prevenção e Controle de Infecção e quatro enfermeiros especialistas em Terapia Intensiva utilizando-se duas rodadas da técnica Delphi. O Índice de Validade de Conteúdo (IVC) do instrumento foi de 0,92 e o coeficiente de confiabilidade alfa de Cronbach foi 0,88, demonstrando que o instrumento é válido e confiável. ${ }^{17}$

Para fins operacionais, foram utilizadas as definições de conhecimento e atitude propostas pela literatura, ${ }^{14}$ em que conhecimento é a conscientização dos profissionais de enfermagem sobre a HB, e atitude é a maneira como os profissionais de enfermagem pensam e se comportam em relação à HB de pacientes críticos. Foi considerado bom conhecimento e boa atitude a taxa de resposta positiva superior ou igual a $70 \%$ (taxa de conhecimento= número de respostas " $3=$ concordo" e " $4=$ concordo totalmente"/total de respostas do questionário de conhecimento X 100; taxa de atitude $=$ número de respostas “ $3=$ concordo" e “ $4=$ concordo totalmente"/total de respostas do questionário de atitude X 100). ${ }^{8}$

A relação dos profissionais de enfermagem das UTIs potencialmente elegíveis, com seus respectivos turnos de trabalho e função, foi obtida junto ao coordenador de enfermagem das unidades. No período do estudo, 146 profissionais de enfermagem atuavam nas UTIs. Os critérios de inclusão foram enfermeiros e técnicos de enfermagem admitidos há 3 meses ou mais e que prestavam assistência direta ao paciente. Foram excluídos profissionais que tinham duplo vínculo (Hospital A e B) no mesmo complexo hospitalar, e também aqueles que estavam de licença médica, férias ou qualquer afastamento de suas atividades laborais.

Os profissionais elegíveis para o estudo foram abordados por uma das pesquisadoras em seus próprios turnos e locais de trabalho. Ao aceitar participar do estudo, o profissional recebeu o 
5 | Felix AMS, Amara IDTA, Soares RAQ, Souza RC.

questionário autoaplicável, sendo orientado a respondê-lo e, em seguida, entregá-lo à pesquisadora. O tempo médio para preenchimento do questionário foi de aproximadamente 15 minutos.

As variáveis do questionário foram codificadas e catalogadas em um dicionário (codebook). Foi elaborada uma base de dados em uma planilha do programa Excel (Microsoft Office 2008 for Mac), com dupla digitação e validação dos dados, a fim de identificar possíveis erros de digitação. As variáveis qualitativas foram descritas por frequências absolutas e porcentagens, enquanto as quantitativas foram descritas por média e desvio-padrão ou medianas e quartis. Para a comparação entre as proporções de concordância e discordância nos dois tipos de instituições, assim como para as demais variáveis qualitativas, foram utilizados testes de qui-quadrado ou testes exatos de Fisher. A idade foi comparada nos grupos por teste t de Student para variâncias homogêneas; as demais variáveis quantitativas foram comparadas por teste não paramétrico de Mann-Whitney. As análises foram realizadas com auxílio do programa SPSS, considerando-se nível de significância de 5\%.

A pesquisa foi aprovada pelo Comitê de Ética da instituição do estudo em 22 de novembro de 2018, sob protocolo $n^{0}$ 3033243. Foram observados todos os preceitos da resolução $n^{0}$ 466/2012 do Conselho Nacional de Saúde sobre pesquisas envolvendo seres humanos. ${ }^{18} \mathrm{O}$ Termo de Consentimento Livre e Esclarecido foi aplicado pelo pesquisador antes do profissional preencher o questionário.

\section{Resultados}

Dos 146 profissionais de enfermagem das UTIs, 65 (44,5\%) não participaram do estudo pelas seguintes razões: $18,4 \%$ se recusaram $(n=12), 15,4 \%$ estavam em férias $(n=10), 15,4 \%$ estavam afastados $(n=$ 10) e $50,8 \%$ trabalhavam no Hospital A e B $(n=33)$. Dos 81 profissionais $(55,5 \%)$ que aceitaram participar do estudo, 63 eram do sexo feminino (77,8\%). O nível de escolaridade mais comum foi o ensino técnico, com $59,2 \%$ dos participantes $(n=48)$; quanto à função, 59 participantes eram técnicos de enfermagem (72,8\%). Os turnos de trabalho foram distribuídos de forma homogênea e, do total de participantes, 17 (21\%) citaram vínculo empregatício em outra instituição (Tabela 1).

A existência de um protocolo sobre HB no setor foi afirmada por $54,3 \%(n=44)$ dos profissionais. Porém, foram observadas diferenças entre as instituições, com taxa maior no Hospital $\mathrm{B}(\mathrm{p}<0,006)$. Isso 
Conhecimento e atitudes de profissionais de enfermagem sobre higiene bucal em... 6

também foi observado quanto à participação em treinamento sobre HB: 46,9\% $(\mathrm{n}=38)$ responderam de forma afirmativa, mas no Hospital B a taxa foi superior $(\mathrm{p}<0,004)$ (Tabela 1).

Tabela 1 - Distribuição dos dados sociodemográficos e profissionais dos participantes do estudo. São Paulo-SP, 2019. ( $\mathrm{n}=81)$

\begin{tabular}{|c|c|c|c|c|c|c|c|}
\hline \multirow{3}{*}{ Variáveis } & \multicolumn{4}{|c|}{ Tipo de instituição } & \multirow{2}{*}{\multicolumn{2}{|c|}{ Total $(n=81)$}} & \multirow{3}{*}{ valor-p } \\
\hline & \multicolumn{2}{|c|}{$\begin{array}{l}\text { Hospital A } \\
\quad(n=59)\end{array}$} & \multicolumn{2}{|c|}{$\begin{array}{c}\text { Hospital B } \\
\quad(n=22)\end{array}$} & & & \\
\hline & $\mathbf{n}$ & $\%$ & $\mathbf{n}$ & $\%$ & $\mathbf{n}$ & $\%$ & \\
\hline Sexo & & & & & & & $0,769^{*}$ \\
\hline Feminino & 45 & 76,3 & 18 & 81,8 & 63 & 77,8 & \\
\hline Masculino & 14 & 23,7 & 4 & 18,2 & 18 & 22,2 & \\
\hline Escolaridade & & & & & & & $>0,99^{*}$ \\
\hline Ensino técnico & 34 & 57,6 & 14 & 63,6 & 48 & 59,2 & \\
\hline Graduação & 13 & 22 & 4 & 18,2 & 17 & 21 & \\
\hline Pós-graduação & 12 & 20,4 & 4 & 18,2 & 16 & 19,7 & \\
\hline Função & & & & & & & $0,298^{*}$ \\
\hline Auxiliar de enfermagem & 7 & 11,9 & 0 & 0 & 7 & 8,7 & \\
\hline Técnico de enfermagem & 42 & 71,1 & 17 & 77,3 & 59 & 72,8 & \\
\hline Enfermeiro & 10 & 17 & 5 & 22,7 & 15 & 18,5 & \\
\hline Turno trabalho & & & & & & & 0,891 \\
\hline Manhã & 21 & 35,6 & 7 & 31,8 & 28 & 34,6 & \\
\hline Tarde & 19 & 32,2 & 8 & 36,4 & 27 & 33,3 & \\
\hline Noite & 19 & 32,2 & 7 & 31,8 & 26 & 32,1 & \\
\hline Vínculo empregatício em outra instituição? & & & & & & & $>0,99^{*}$ \\
\hline $\operatorname{Sim}$ & 12 & 20,3 & 5 & 22,7 & 17 & 21 & \\
\hline Não & 47 & 79,7 & 17 & 77,3 & 64 & 79 & \\
\hline Protocolo/rotina sobre $\mathrm{HB}^{+}$? & & & & & & & $<0,006^{*}$ \\
\hline $\operatorname{Sim}$ & 28 & 47,4 & 16 & 72,7 & 44 & 54,3 & \\
\hline Não & 27 & 45,8 & 3 & 14,0 & 30 & 37 & \\
\hline Não sei & 4 & 6,8 & 3 & 14,0 & 7 & 8,7 & \\
\hline Treinamento sobre $\mathrm{HB}^{+}$ & & & & & & & $<0,004$ \\
\hline Sim & 22 & 37,3 & 16 & 72,7 & 38 & 46,9 & \\
\hline Não & 37 & 62,7 & 6 & 27,3 & 43 & 53,1 & \\
\hline
\end{tabular}

A idade média dos participantes foi de 36,6 anos (entre 20 e 58 anos), o tempo médio de atuação profissional foi de 7 anos $(0,3$ - 30) e o tempo médio de atuação em UTI foi de 6 anos (0,3 - 25). Referente à carga horária semanal de trabalho, a média foi de 37,4 horas $(30$ - 48) (Tabela 2). 
Tabela 2 - Valor da média, desvio-padrão, valores mínimos e máximos, e valor de p das variáveis idade, anos de experiência, anos de experiência em UTI e carga horária semanal dos participantes do estudo. São Paulo-SP, 2019. $(n=81)$

\begin{tabular}{|c|c|c|c|c|c|c|c|c|c|c|}
\hline \multirow{3}{*}{ Variável } & \multicolumn{6}{|c|}{$\begin{array}{l}\text { Tipo de instituição } \\
\end{array}$} & & & & \multirow{3}{*}{ valor-p } \\
\hline & \multicolumn{3}{|c|}{ Pública $(n=59)$} & \multicolumn{3}{|c|}{ Privada $(n=22)$} & \multicolumn{3}{|c|}{ Total $(n=81)$} & \\
\hline & Média & $\mathrm{DP}$ & mín-max & Média & DP & mín-máx & Média & DP & mín-máx & \\
\hline Idade (anos) & 37,1 & 8,5 & $20-58$ & 35,5 & 9,3 & $21-53$ & 35,5 & 9,3 & $20-58$ & $0,470^{*}$ \\
\hline Anos de experiência & 7 & 6 & $0,3-30$ & 7 & 7 & $0,3-30$ & 7 & 7 & $0,3-30$ & $0,646^{+}$ \\
\hline $\begin{array}{l}\text { Anos de experiência em } \\
\text { UTI }\end{array}$ & 6 & 7 & $0,3-25$ & 5 & 5 & $0,3-18$ & 5 & 5 & $0,3-25$ & $0,392^{+}$ \\
\hline $\begin{array}{l}\text { Carga horária semanal } \\
\text { (horas) }\end{array}$ & 37 & 3,8 & $30-48$ & 37,6 & 4,4 & $33-48$ & 37,6 & 4,4 & $30-48$ & $0,880^{+}$ \\
\hline
\end{tabular}

Notas: DP: Desvio Padrão; mín: mínimo, máx: máximo; UTI= Unidade de Terapia Intensiva. ${ }^{\star}$ Teste t de Student, 'Teste de Mann-Whitney

Com relação aos conhecimentos e atitudes sobre $\mathrm{HB}$ de pacientes críticos, as categorias “concordo" e "concordo totalmente", e “discordo" e discordo totalmente”, foram agrupadas para possibilitar a comparação entre as diferentes instituições (Tabela 3 e 4).

Ao analisar a taxa de respostas positivas referentes aos conhecimentos e atitudes sobre HB, constatou-se que a taxa de conhecimento dos profissionais do Hospital A foi de 76,6\% (588 respostas “concordo" e "concordo totalmente"/767 respostas), enquanto daqueles do Hospital B foi de 81,8\% (234 respostas “concordo" e "concordo totalmente"/286 respostas). Não foram identificadas diferenças estatísticas significativas entre profissionais do Hospital A e B quanto aos conhecimentos sobre HB (Tabela 3).

Acerca das atitudes, no Hospital A a taxa de respostas positivas foi de 62,7\% (481 respostas "concordo" e "concordo totalmente"/767 respostas) e no Hospital B foi de 67,1\% (192 respostas “concordo" e "concordo totalmente"/286 respostas). Houve diferença estatística significativa quanto ao uso de escova de dentes com cerdas macias e sucção, em que 15,3\% (n= 9) dos participantes do Hospital A e 54,5\% ( $n=12)$ dos participantes do Hospital B concordaram com essa afirmativa $(p<0,001)$ (Tabela 4). 
Conhecimento e atitudes de profissionais de enfermagem sobre higiene bucal em... 8

Tabela 3 - Distribuição das respostas dos participantes do estudo segundo conhecimento sobre HB de pacientes críticos. São Paulo-SP, 2019. ( $\mathrm{n}=81)$

Tipo de instituição

\begin{tabular}{|c|c|c|c|c|c|c|c|c|c|}
\hline \multirow{3}{*}{ Afirmativas } & \multicolumn{4}{|c|}{ Hospital A $(n=59)$} & \multicolumn{4}{|c|}{ Hospital B $(n=22)$} & \multirow{3}{*}{ valor-p } \\
\hline & \multicolumn{2}{|c|}{$\begin{array}{l}\text { Concordo e } \\
\text { Concordo } \\
\text { totalmente }\end{array}$} & \multicolumn{2}{|c|}{$\begin{array}{c}\text { Discordo e } \\
\text { Discordo } \\
\text { totalmente }\end{array}$} & \multicolumn{2}{|c|}{$\begin{array}{l}\text { Concordo e } \\
\text { Concordo } \\
\text { totalmente }\end{array}$} & \multicolumn{2}{|c|}{$\begin{array}{l}\text { Discordo e } \\
\text { discordo } \\
\text { totalmente }\end{array}$} & \\
\hline & $\mathrm{n}$ & $\%$ & $\mathbf{n}$ & $\%$ & $\mathbf{n}$ & $\%$ & $\mathrm{n}$ & $\%$ & \\
\hline $\begin{array}{l}\text { 1. A PAVM } \text { P }^{\dagger} \text { pode ser } \\
\text { prevenida } \\
\text { estratégias } \\
\text { incluem a } \mathrm{HB} \text {. }\end{array}$ & 57 & 96,6 & 2 & 3,4 & 21 & 95,5 & 1 & 4,5 & $>0,99^{*}$ \\
\hline $\begin{array}{l}\text { 2. A } \mathrm{HB}^{+} \text {deve ser } \\
\text { realizada pelo menos } \\
\text { uma vez a cada } \\
\text { plantão. }\end{array}$ & 56 & 94,9 & 3 & 5,1 & 19 & 86,4 & 3 & 13,6 & $0,337^{*}$ \\
\hline $\begin{array}{l}\text { 3. A } \mathrm{HB}^{+} \text {inclui a } \\
\text { escovação } \\
\text { dentes/ próteses. }\end{array}$ & 57 & 96,6 & 2 & 3,4 & 22 & 100 & 0 & 0,0 & $>0,99^{*}$ \\
\hline $\begin{array}{l}\text { 4. } A \mathrm{HB}^{*} \text { inclui as } \\
\text { gengivas. }\end{array}$ & 58 & 98,3 & 1 & 1,7 & 22 & 100 & 0 & 0,0 & $>0,99^{*}$ \\
\hline $\begin{array}{l}\text { 5. } \mathrm{A} \mathrm{HB}^{*} \text { inclui a } \\
\text { língua. }\end{array}$ & 58 & 98,3 & 1 & 1,7 & 22 & 100 & 0 & 0,0 & $>0,99^{*}$ \\
\hline $\begin{array}{l}\text { 6. A } \mathrm{HB}^{+} \text {inclui a } \\
\text { hidratação } \mathrm{da} \\
\text { mucosa peribucal e } \\
\text { bucal. }\end{array}$ & 57 & 96,6 & 2 & 3,4 & 22 & 100 & 0 & 0,0 & $>0,99^{*}$ \\
\hline $\begin{array}{l}\text { 7. A } \mathrm{HB}^{+} \text {inclui a } \\
\text { aferição da pressão } \\
\text { de cuff antes do } \\
\text { procedimento. }\end{array}$ & 42 & 72,2 & 19 & 32,2 & 18 & 81,8 & 4 & 18,2 & 0,222 \\
\hline $\begin{array}{l}\text { 8. A } \mathrm{HB}^{*} \text { inclui a } \\
\text { aferição da pressão } \\
\text { de cuff após o } \\
\text { procedimento. }\end{array}$ & 50 & 84,7 & 9 & 15,3 & 19 & 86,4 & 3 & 13,6 & $>0,99^{*}$ \\
\hline $\begin{array}{l}\text { 9. A } \mathrm{HB}^{*} \text { inclui a } \\
\text { aferição da posição } \\
\text { de tubo antes do } \\
\text { procedimento. }\end{array}$ & 45 & 76,3 & 14 & 23,7 & 19 & 86,4 & 3 & 13,6 & $0,214^{*}$ \\
\hline $\begin{array}{l}\text { 10. A } \mathrm{HB}^{*} \text { inclui a } \\
\text { aferição da posição } \\
\text { de tubo após o } \\
\text { procedimento }\end{array}$ & 40 & 67,8 & 19 & 32,2 & 19 & 86,4 & 3 & 13,6 & 0,114 \\
\hline $\begin{array}{l}\text { 11. A } \mathrm{HB}^{t} \text { inclui a } \\
\text { aspiração subglótica. }\end{array}$ & 46 & 78,0 & 13 & 22 & 19 & 86,4 & 3 & 13,6 & $0,331^{*}$ \\
\hline
\end{tabular}

Rev. Enferm. UFSM, Santa Maria, v11, p. 1-17, 2021 
9 | Felix AMS, Amara IDTA, Soares RAQ, Souza RC.

12. $\mathrm{Na}$ minha

unidade, a avaliação

$\begin{array}{llllllllll}\text { da cavidade oral é } & 19 & 32,2 & 40 & 67,8 & 12 & 54,5 & 10 & 45,5 & 0,092\end{array}$

feita até $72 \mathrm{~h}$ após a

admissão na $\mathrm{UTI}^{\S}$.

13. $\mathrm{Na}$ minha

unidade, a avaliação

da cavidade oral é $\quad \begin{array}{lllllllll}5,1 & 56 & 94,9 & 0 & 0 & 22 & 100,0 & 0,565^{*}\end{array}$

feita por cirurgião-

dentista.

Notas: " comparação com teste exato de Fisher. Demais casos comparados com teste de qui-quadrado. ${ }^{+} \mathrm{PAVM}=$ pneumonia associada à ventilação mecânica; $\mathrm{HB}=$ higiene bucal; ${ }^{\S} \mathrm{UTI}=$ Unidade de Terapia Intensiva

Tabela 4 - Distribuição das respostas dos participantes do estudo segundo atitudes sobre HB de pacientes críticos. São Paulo-SP, 2019. (n=81)

Tipo de instituição

Hospital A ( $\mathbf{n}=59)$

Hospital B ( $n=22)$

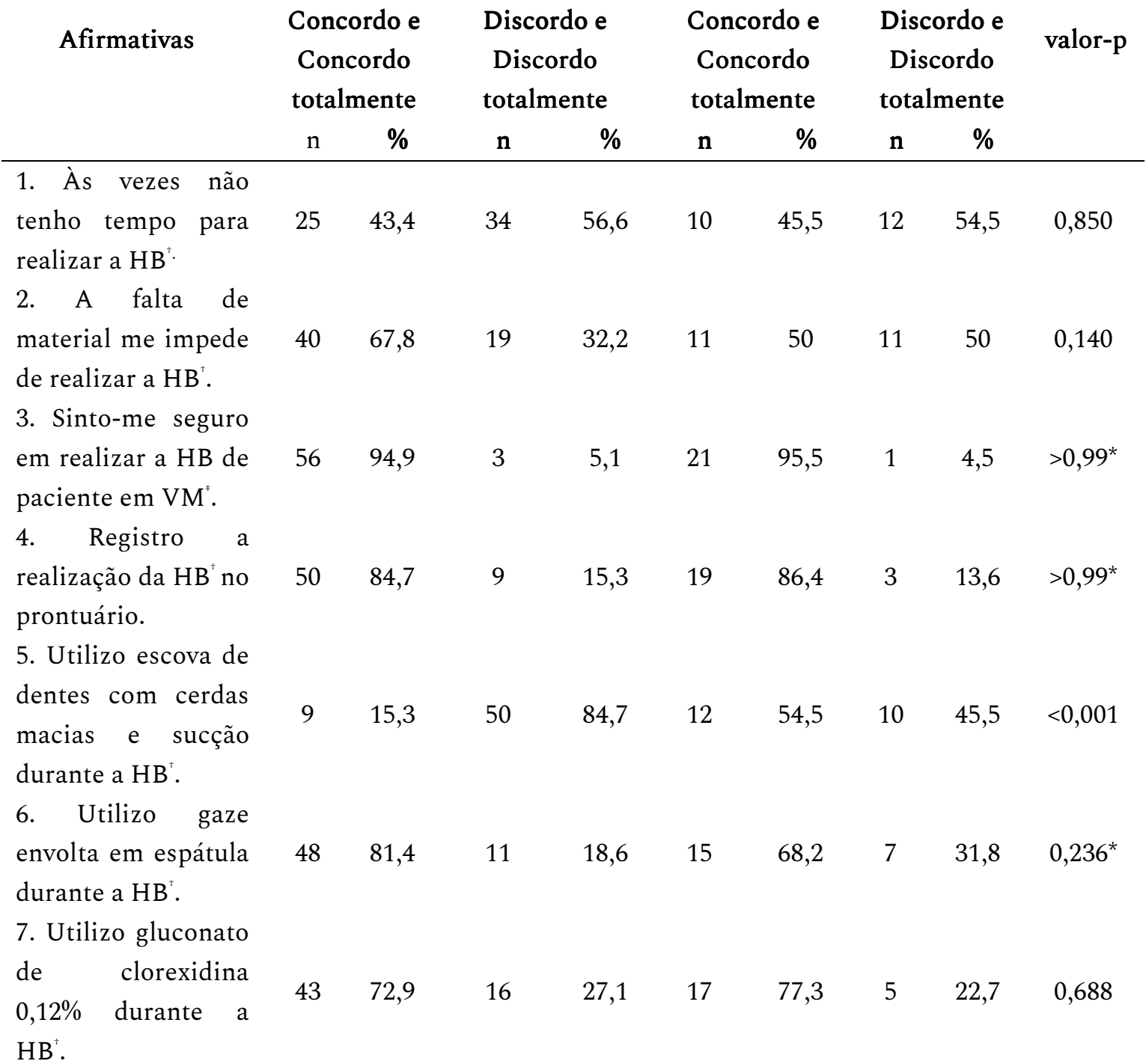


Conhecimento e atitudes de profissionais de enfermagem sobre higiene bucal em... $\mid 10$

\begin{tabular}{|c|c|c|c|c|c|c|c|c|c|}
\hline \multicolumn{10}{|l|}{ 8. Faço a hidratação } \\
\hline $\begin{array}{l}\text { da mucosa } \\
\text { peribucal e bucal } \\
\text { após a } \mathrm{HB}^{+} \text {. }\end{array}$ & 56 & 94,9 & 3 & 5,1 & 22 & 100 & 0 & $0,0 \%$ & $0,563^{*}$ \\
\hline 9. Faço a aferição da & & & & & & & & & \\
\hline $\begin{array}{l}\text { pressão do cuff } \\
\text { antes da } \mathrm{HB}^{+} .\end{array}$ & 21 & 35,6 & 38 & 64,4 & 7 & 31,8 & 15 & 68,2 & 0,822 \\
\hline $\begin{array}{l}\text { 10. Faço a aferição } \\
\text { da pressão do cuff } \\
\text { após a } \mathrm{HB}^{+} \text {. }\end{array}$ & 23 & 39 & 36 & 61 & 6 & 27,3 & 16 & 72,7 & 0,394 \\
\hline $\begin{array}{l}\text { 11. Faço a aferição } \\
\text { da posição do tubo } \\
\text { antes da } \mathrm{HB}^{\dagger} \text {. }\end{array}$ & 38 & 64,4 & 21 & 35,6 & 19 & 86,4 & 3 & 13,6 & 0,054 \\
\hline $\begin{array}{l}\text { 12. Faço a aferição } \\
\text { da posição do tubo } \\
\text { após a } \mathrm{HB}^{+} \text {. }\end{array}$ & 40 & 67,8 & 19 & 32,2 & 19 & 86,4 & 3 & 13,6 & 0,114 \\
\hline $\begin{array}{l}\text { 13. Faço a aspiração } \\
\text { subglótica durante a }\end{array}$ & 32 & 54,2 & 27 & 45,8 & 14 & 63,6 & 8 & 36,4 & 0,217 \\
\hline $\mathrm{HB}^{+}$ & & 34,2 & & & 17 & & 0 & $30, \mathrm{~T}$ & 0,217 \\
\hline
\end{tabular}

Notas: ${ }^{*}$ comparação com teste exato de Fisher. Demais casos comparados com teste de qui-quadrado; ${ }^{\dagger} \mathrm{HB}=$ higiene bucal; ${ }^{*} \mathrm{VM}=$ ventilação mecânica

\section{Discussão}

Há divergência na literatura sobre associações significativas entre as características demográficas, profissionais e as atitudes em relação à $\mathrm{HB}$ de pacientes críticos, com estudo que não confirma essa associação ${ }^{5}$ e estudo que descreve que o nível de escolaridade, a experiência profissional e o treinamento em HB são fatores relevantes na atitude dos enfermeiros. ${ }^{14}$

Neste estudo, os participantes apresentam perfil sociodemográfico e profissional semelhante a estudos prévios em que a maioria era do sexo feminino, ${ }^{3,5-8,10,13-14}$ com idade aproximada de 37 anos, ${ }^{5}$ ensino médio, ${ }^{6}$ experiência profissional menor que seis anos ${ }^{10,14}$ e experiência em UTI de quatro anos; ${ }^{9}$ e reconhecem a importância da HB ao afirmarem que a PAVM pode ser prevenida com estratégias que incluem a HB; esses resultados são condizentes com a literatura sobre o assunto..$^{6-8,10,13-14}$

A execução da HB sofre a interferência de fatores individuais, por exemplo, o nível de conhecimento e tempo de experiência profissional, e fatores organizacionais, como a oferta de treinamento, o número suficiente de profissionais e insumos adequados. ${ }^{19}$ Sendo assim, conhecer esses 
11 | Felix AMS, Amara IDTA, Soares RAQ, Souza RC.

fatores ajuda a identificar barreiras e a traçar estratégias corretivas que podem melhorar os conhecimentos, as atitudes e a prática de HB em pacientes críticos.

Acerca da presença de protocolos sobre HB, houve diferença significativa entre as instituições, sendo que esse aspecto foi mais afirmado pelos profissionais do Hospital B. Pesquisas realizadas em hospitais na Índia e Arábia Saudita ${ }^{10}$ apontam que a adesão ao protocolo de HB foi em média de 65\%.

Protocolos assistenciais são úteis na área da enfermagem. Trata-se de uma ferramenta baseada em evidência científica que ajuda a diminuir variações no cuidado prestado ao paciente, proporcionando maior satisfação para a equipe de enfermagem, maior segurança para o paciente, além de ter finalidade educativa. ${ }^{20}$

Com relação à participação em treinamentos sobre HB, nota-se uma diferença significativa entre as instituições, sendo que o Hospital B apresentou melhor desempenho. Tal achado merece atenção, pois sugere que o conhecimento sobre HB é obtido de forma empírica, o que pode resultar em variações no cuidado e práticas inseguras.

A educação permanente é fator determinante para aumentar o arcabouço teórico dos profissionais e a adesão às boas práticas. ${ }^{16}$ Além disso, ela está prevista na Resolução da Diretoria Colegiada (RDC) no 36 como estratégia para reforçar as práticas de segurança do paciente. ${ }^{21}$ Posto isso, os serviços de saúde precisam fornecer treinamentos articulados ao processo de trabalho, em quantidade suficiente e em intervalos regulares para desenvolver e manter a competência dos seus colaboradores. É essencial que os profissionais de saúde estejam cientes e atualizados sobre as melhores práticas, demonstrando aos usuários do serviço que eles estão trabalhando continuamente para garantir que altos padrões de atendimento sejam prestados.

A prevenção de PAVM é um objetivo comum dos profissionais que atuam em UTI, independentemente do serviço ser público ou privado. Logo, eles precisam entender a fisiopatogenia da PAVM e o papel das medidas de prevenção - como exemplo, a HB - na prevenção da transmissão de microrganismos patogênicos. As capacitações sobre prevenção desse tipo de pneumonia podem ser ministradas em parceria entre profissionais do Serviço de Controle de Infecção, Educação Permanente, UTI, e os conteúdos devem ser focados nas necessidades dos profissionais. Como estratégias de ensino- 
Conhecimento e atitudes de profissionais de enfermagem sobre higiene bucal em... $\mid 12$

aprendizagem para esse conteúdo, sugere-se aquelas que possibilitam a aprendizagem significativa, tais como a aprendizagem baseada em problemas (ABP) e simulação realística. ${ }^{22}$

A literatura internacional aponta que as taxas de conhecimento sobre $\mathrm{HB}$ variam de $31,8 \%$ a $97,4 \%,,^{3-4,7-10,14,16}$ enquanto que as atitudes e práticas variam de $57 \%$ a $91 \% .^{4-5,8,10,14,16}$ No presente estudo, ambos hospitais apresentaram níveis adequados de conhecimento e atitudes frágeis em relação à HB. Quando comparados, a taxa de conhecimento e atitudes autorreferidas pelos profissionais do Hospital B foram superiores às dos profissionais do Hospital A.

Chama atenção o fato de que mesmo com conhecimento adequado, profissionais de ambos hospitais apresentaram baixas atitudes, o que pode sugerir fragilidades na cultura de segurança do paciente nas instituições estudadas. A cultura de segurança do paciente é o conjunto de valores, competências e padrões de comportamento que determinam o comprometimento do serviço com a gestão da saúde e segurança de seus pacientes por meio da implantação de práticas seguras e diminuição de incidentes. ${ }^{21}$ Nesse contexto, os gestores dos serviços de saúde se encontram em melhor posição para induzir mudanças nas atitudes e práticas dos profissionais, podendo contribuir para o estabelecimento da cultura da segurança do paciente como um pré-requisito da assistência. ${ }^{23}$

Neste estudo, aproximadamente metade dos profissionais do Hospital B conhece e realiza a avaliação da cavidade oral dentro das primeiras $72 \mathrm{~h}$ da admissão do paciente na UTI, enquanto menos da metade dos participantes do Hospital A conhece e aplica essa recomendação.

A avaliação adequada da saúde bucal e a intervenção precoce geralmente impedem complicações graves antes que essas possam comprometer os resultados terapêticos. ${ }^{1}$ Por meio do processo de enfermagem, os profissionais são capazes de reconhecer e intervir precocemente em mudanças nas necessidades humanas básicas dos pacientes.

Segundo o guia de recomendação da Agência Nacional de Vigilância Sanitária (ANVISA), a colonização da cavidade oral de pacientes em uso de ventilação mecânica por microrganismos Gram negativos tende a ocorrer entre 48 a 72h após a intubação e, não raro, ocorrem complicações periodontais, periapicais e mucosites. ${ }^{1}$ Assim, a avaliação da cavidade oral é relevante para o cuidado desses pacientes.

A presença de um cirurgião-dentista não foi considerada importante pelos participantes de 
ambos hospitais, contrapondo estudos realizados na Etiópia e no Brasil, para os quais a presença desse profissional foi considerada relevante pela equipe de enfermagem devido ao auxílio no diagnóstico, tratamento de complicações e orientação quanto à técnica adequada da HB. ${ }^{13-14}$ Contudo, independentemente da prática colaborativa interdisciplinar - um dos pilares do cuidado centrado na pessoa, o enfermeiro pode e tem condições de assumir a liderança nesse processo. ${ }^{24}$

Estudos descrevem várias barreiras para a realização da HB, sendo as mais relevantes a falta de tempo, escassez de materiais, ${ }^{3-5,7,9,25}$ proporção inadequada entre enfermeiros e pacientes, ${ }^{6,8,12}$ falta de cooperação do paciente, ${ }^{4}$ lacunas de treinamento, ${ }^{3,10}$ percepção do procedimento como desagradável, ${ }^{10,25} \mathrm{e}$ receio de deslocar o tubo endotraqueal. ${ }^{9}$ No presente estudo, a falta de tempo e de materiais foram as mais expressivas em ambos hospitais.

Quanto aos materiais utilizados para $\mathrm{HB}$, houve diferença significativa entre as instituições quanto ao uso de escova de dentes de cerdas macias e sucção para a HB. Semelhante a estudos prévios, ${ }^{6,10,13}$ o uso desse material ainda não está totalmente implantado nos serviços, mostrando que os profissionais de enfermagem realizam cuidados bucais que não são baseados em evidência. ${ }^{1}$

Em relação ao registro do procedimento no prontuário do paciente, a presente pesquisa aponta que os profissionais de enfermagem de ambos hospitais adotam essa prática - resultado oposto ao encontrado na literatura, em que a taxa de registro variou entre $46 \%$ a $73,8 \% .^{6,8,26}$ Vale ressaltar que esse aspecto está dentre as melhores práticas assistenciais e a sua adoção reflete a qualidade do cuidado.

Em concordância com estudo prévio, ${ }^{8}$ nota-se que grande parte dos profissionais de enfermagem dos Hospitais A e B não implementa a recomendação de avaliar o cuffantes e após a HB e nem fazem a aspiração subglótica conforme prática fortemente recomendada por guia nacional, ${ }^{1}$ reforçando a necessidade de protocolo baseado em evidência e o fortalecimento da cultura de segurança nos hospitais estudados.

Este estudo apresentou limitações, como o uso de um questionário autoaplicável, que apesar de ser considerado válido e confiável pelos especialistas, não reflete as reais circunstâncias em que foi respondido, pois a prática profissional não foi avaliada. Outras limitações se referem à comparação entre instituições com realidades diferentes e também ao delineamento do estudo, o que compromete a validade externa, permitindo identificar conhecimentos e atitudes apenas em um determinado recorte temporal. 
Conhecimento e atitudes de profissionais de enfermagem sobre higiene bucal em... $\mid 14$

\section{Conclusão}

Os profissionais de enfermagem das UTIs estudadas possuem conhecimento adequado e atitudes frágeis sobre a HB de pacientes críticos. Profissionais do Hospital B apresentaram melhor taxa de conhecimento e atitudes quando comparados aos profissionais do Hospital A.

A participação em treinamentos, a presença de protocolo de HB e o uso de escova de dentes com cerdas macias e sucção foram estatisticamente significantes entre as instituições, sendo que os profissionais de enfermagem do Hospital B apresentaram mais respostas afirmativas a esses aspectos.

É recomendável que as UTIs reforcem a cultura de segurança do paciente por meio da implantação de diretrizes baseadas em evidências científicas sobre HB, oferta periódica de programas de educação permanente e disponibilização de insumos apropriados para que os profissionais possam realizar a HB de forma efetiva.

\section{Referências}

1. Agência Nacional de Vigilância Sanitária (BR). Medidas de prevenção de infecção relacionada à assistência à saúde [Internet]. Brasília (DF): Anvisa; 2017 [acesso em 2020 abr 08]. Disponível em: https://www20.anvisa.gov.br/segurancadopaciente/index.php/publicacoes/item/caderno-5

2. Torres A, Niederman MS, Chastre J, Ewig S, Fernandez-Vandellos P, Handerger H, et al. International ERS/ESICM/ESCMID/ALAT guidelines for the management of hospital-acquired pneumonia and ventilator-associated pneumonia. Eur Respir J. 2017 Sept;50(3):1700582. doi: https://dx.doi.org/10.1183/13993003.00582-2017

3. Nagarakanti S, Avuluri J, Chava VK. Evaluation of nurse's attitude toward the provision of oral hygiene care to hospitalized patients at two private hospitals in South India. Iran J Nurs Midwifery Res. 2019;24(6):472-74. doi: https://dx.doi.org/10.4103/ijnmr.IJNMR_39_19

4. Lin YS, Chang JC, Chang TH, Lou MF. Critical care nurses' knowledge, attitudes and practices of oral care for patients with oral endotracheal intubation: a questionnaire survey. J Clin Nurs. 2011 Nov;20(2122):3204-14. doi: https://doi.org/10.1111/j.1365-2702.2011.03819.x

5. Saddki N, Sani FEM, Tin-Oo MM. Oral care for intubated patients: a survey of intensive care unit nurses. Nurs Crit Care. 2017;22(2):89-98. doi: https://doi.org/10.1111/nicc.12119

6. Rodrigues SS, Caminha MFC, Ferraz MGG, Arruda MA, Kozmhinshy VMR, Guerra CARM, et al. 
15 | Felix AMS, Amara IDTA, Soares RAQ, Souza RC.

Knowledge, attitude and practice of the nursing team regarding oral health care in intensive care units in a reference hospital of Recife, Brazil. Pesqui Bras Odontopediatria Clín Integr. 2016;16(1):129-39. doi: https://doi.org/10.4034/PBOCI.2016.161.14

7. Aboalizm SE, Kasemy ZAA. Nurse's knowledge, attitude and practice toward mouth hygiene among critical ill patients. Int J Novel Res Healthc Nurs [Internet]. 2016 [cited 2020 Apr 10];3(3):1-15. Available from: https://www.noveltyjournals.com/issue/IJNRHN/Issue-3-September-2016-December-2016

8. Asia M, Muhammad NA, Hajra S, Wagas A, Sarwar H, et al. Knowledge, attitude and practices of nurses to oral care to hospitalized patients in services hospital Labore. Saudi J Med Pharm Sci. 2017;3(5):399-407. doi: https://doi.org/10.21276/sjmps

9. Sreenivasan VPD, Ganganna A, Rajashekaraiah PB. Awareness among intensive care nurses regarding oral care in critically ill patients. J Indian Soc Periodontol. 2018 Nov-Dec;22(6):541-45. doi: https://doi.org/10.4103/jisp.jisp_30_18

10. Alja'afreh MA, Mosleh SM, Habashneh SS. Nurses' perception and attitudes towards oral care practices for mechanically ventilated patients. Saudi Med J. 2018;39(4):379-85. doi: https://doi.org/10.15537/smj.2018.4.21749

11. Dagnew ZA, Abraham IA, Beraki GG, Tesfamariam EH, Mittler S, Tesfamichael YZ. Nurses' attitude towards oral care and their practicing level for hospitalized patients in Orotta National Referral Hospital, Asmara-Eritrea: a cross-sectional study. BMC Nurs. 2020;19(63). doi: https://doi.org/10.1186/s12912-020-00457-3

12. Oh E, Choi JS. Factors influencing the adherence of nurses to standard precautions in South Korea hospital settings. Am J Infect Control. 2019;47(11):1346-51. doi: https://doi.org/10.1016/j.ajic.2019.05.015

13. Silva DP, Silva MS, Silva DP, Ferraz MAAL, Falcão CAM, Moura CDVS. Práticas de higiene bucal aplicadas em pacientes internados em Unidades de Terapia Intensiva. Rev Ciênc Saberes [Internet]. 2018 [acesso em 2020 maio 10];4(1):815-19. Disponível em: http://www.facema.edu.br/ojs/index.php/ReOnFacema/article/view/368/185

14. Andargie ST, Kassahun CW. Knowledge and attitude of nurses' towards patient's oral care at University of Gondar comprehensive specialized hospital, Northwest Ethiopia. Int J Afr Nurs Sci. 2019;11:100165. doi: https://doi.org/10.1016/j.ijans.2019.100165

15. Tanguay A, Reeves I, Lemay S, Khadra C, Gosselin E, St-Cyr-Tribble D. Survey of oral care practices in Quebec for intensive care patients receiving mechanical ventilation. Can J Crit Care Nurs [Internet]. 2018 [cited 2020 Jun 19];29(3):39-44. Available from: https://www.caccn.ca/files/CJCCN/29-32018\%20CJCCN.pdf\#page=39

16. Tembo E. Intensive care nurses' knowledge, attitudes and practices of oral care for patients with oral endotracheal intubation [Internet]. Johannesburg: University of the Witwatersrand, Faculty of Health 
Conhecimento e atitudes de profissionais de enfermagem sobre higiene bucal em... $\mid 16$

Sciences; 2016 [cited 2020 May 04]. Available from: https://pdfs.semanticscholar.org/f96f/3624167d593302393ce7b9da2403e0c19b82.pdf?_ga=2.101741057.1695 240205.1587994223-292557316.1557689665

17. Souza AC, Alexandre NMC, Guirardello EB. Propriedades psicométricas na avaliação de instrumentos: avaliação da confiabilidade e da validade. Epidemiol Serv Saúde. 2017;26(3):649-59. doi: https://doi.org/10.5123/s1679-49742017000300022

18. CONSELHO NACIONAL DE SAÚDE. Resolução nº 466, de 12 de dezembro de 2012. Diretrizes e normas regulamentadoras de pesquisas envolvendo seres humanos. Brasília, DF, 2013. Disponível em: http://conselho.saude.gov.br/resolucoes/2012/Reso466.pdf. Acesso em: 30 maio 2020.

19. Ciampoli N, Bouchoucha S, Currey J, Hutchinson A. Evaluation of prevention of ventilator-associated infections in four Australian intensive care units. J Infect Prev. 2020 May;21(4):147-54. doi: $10.1177 / 1757177420908006$

20. Sales CB, Bernardes A, Gabriel CS, Brito MFP, Moura AA, Zanetti ACB. Protocolos Operacionais Padrão na prática profissional da enfermagem: utilização, fragilidades e potencialidades. Rev Bras Enferm. 2018 Feb;71(1):126-34. doi: https://doi.org/10.1590/0034-7167-2016-0621

21. BRASIL. Ministério da Saúde. Agência Nacional de Vigilância Sanitária. Resolução da Diretoria Colegiada (RDC) no 36, de 25 de julho de 2013. Institui ações para a segurança do paciente em serviços de saúde e dá outras providências. Brasília, DF: Ministério da Saúde, 2013. Disponível em: https://bvsms.saude.gov.br/bvs/saudelegis/anvisa/2013/rdc0036_25_07_2013.html. Acesso em: 23 jul. 2020.

22. Felix AMS, Soares RAQ. Metodologias ativas no ensino de enfermagem em doenças transmissíveis. Rev Enferm UFPE On Line. 2019;13:e241816 doi: https://doi.org/10.5205/1981-8963.2019.241816

23. Andrade LEL, Lopes JM, Souza Filho MCM, Vieira Júnior RF, Farias LPC, Santos CCM, et al. Cultura de segurança do paciente em três hospitais brasileiros com diferentes tipos de gestão. Ciênc Saúde Colet. 2018 jan;23(1):161-72. doi: https://doi.org/10.1590/1413-81232018231.24392015

24. Harnagea H, Couturier Y, Shrivastava R, Girad F, Lamothe L, Bedos CP, et al. Barriers and facilitators in the integration of oral health into primary care: a scoping review. BMJ Open. 2017;7(9):e016078. doi: https://doi.org/10.1136/bmjopen-2017-016078

25. Tanguay A, LeMay S, Reeves I, Gosselin E, St-Cyr-Tribble D. Factors influencing oral care in intubated intensive care patients. Nurs Crit Care. 2019 Jul;25(1):53-60. doi: https://doi.org/10.1111/nicc.12456

26. Ashour AA. Knowledge, attitudes and practices regarding oral health and oral care among nursing staff at a mental health hospital in Taif, Saudi Arabia: a questionnaire-based study. J Adv Oral Res. 2020 May;11(1):34-44. doi: https://doi.org/10.1177/2320206820910300 
17 | Felix AMS, Amara IDTA, Soares RAQ, Souza RC.

Editor Científico: Tânia Solange Bosi de Souza Magnago

Editor Associado: Rhanna Emanuela Fontenele Lima de Carvalho

\section{Autor correspondente.}

Adriana Maria da Silva Felix

E-mail: adriana.felix@fcmsantacasasp.edu.br

Endereço: Rua Doutor Cesário Motta Júnior, 61.

CEP: 01221-020

\section{Contribuições de Autoria}

\section{1 - Adriana Maria da Silva Felix}

Concepção ou desenho do estudo/pesquisa, análise e/ou interpretação dos dados, revisão final com participação crítica e intelectual no manuscrito

\section{2 - Isabela Di Tilia Alves do Amara}

Concepção ou desenho do estudo/pesquisa, análise e/ou interpretação dos dados, revisão final com participação crítica e intelectual no manuscrito

\section{3 - Rosemeire Ângela de Queiroz Soares}

Análise e/ou interpretação dos dados, revisão final com participação crítica e intelectual no manuscrito

\section{4 -Regina Claudia de Souza}

Análise e/ou interpretação dos dados, revisão final com participação crítica e intelectual no manuscrito

\section{Como citar este artigo}

Felix AMS, Amara IDTA, Souza RC, Soares RAQ. Conhecimento e atitudes de profissionais de enfermagem sobre higiene bucal em pacientes críticos. Rev. Enferm. UFSM. 2021 [Acesso em: Ano Mês Dia]; vol.11 e18: 1-17. DOI:https://doi.org/10.5902/2179769247245 